\title{
El tema de la evolución biológica en los libros de texto de la escuela socialista en México (1930-1940)*
}

The Topic of Evolution in Mexican Socialist School (1930-1940)

Alicia Villela ${ }^{\dagger}$

Erica Torrens ${ }^{\ddagger}$

Ana Barahona ${ }^{\text {s+† }}$

\begin{abstract}
Resumen
El tema de la evolución de los seres vivos resulta fundamental tanto para la biología como para la educación científica. En este trabajo se presenta una parte de un proyecto de investigación mayor que busca analizar tanto la presencia, como el estado de la teoría de la evolución biológica en los libros de texto de educación básica mexicanos, desde que se creó la Secretaría de Educación Pública en 1921.

El análisis histórico y de los contenidos del tema de la evolución biológica en los libros de texto mexicanos ha sido poco tratado. El presente texto se enfoca en un periodo interesante y de particular importancia en la historia educativa de México: el proyecto de la educación socialista, que fue propuesto e implementado brevemente durante la década de 1930. Las autoras ponen particular énfasis en la ideología socialista detrás de la enseñanza de la evolución, puesto que es lo distintivo del periodo y del proyecto.
\end{abstract}

Palabras clave: evolución - educación - libros de texto - escuela socialista

\begin{abstract}
The topic of biological evolution is fundamental to both biology and science education. This paper advances some results of a broad research project that seeks to analyse both the presence and the state of the theory of evolution in Mexican textbooks of basic education, since the creation of the Ministry of Public Education in 1921, under a historical and philosophical perspective.

The study of the contents of evolution in textbooks from the history and philosophy of science has been little studied in Mexico. This paper focuses on an interesting period of particular importance in the educational history of Mexico: the project of socialist education, which was proposed and implemented briefly during the 1930s.
\end{abstract}

Keywords: evolution - education - textbooks - socialist education

* Recibido: 7 de Marzo de 2016. Aceptado con revisiones: 5 de Diciembre de 2016.

${ }^{\dagger}$ Grupo de Estudios Sociales de la Ciencia, Departamento de Biología Evolutiva, Facultad de Ciencias, Universidad Nacional Autónoma de México.

* Grupo de Estudios Sociales de la Ciencia, Departamento de Biología Evolutiva, Facultad de Ciencias, Universidad Nacional Autónoma de México. Para contactar a las autoras, por favor, escribir a: torrens@ciencias.unam.mx.

$\$$ Grupo de Estudios Sociales de la Ciencia, Departamento de Biología Evolutiva, Facultad de Ciencias, Universidad Nacional Autónoma de México.

${ }^{\dagger \dagger}$ Esta investigación no hubiera sido posible sin el apoyo de los proyectos UNAM PAPIIT IN404116 y CONACYT CB2012-178031-H. Metatheoria 8(2)(2018): 169-180. ISSN 1853-2322.

(c) Editorial de la Universidad Nacional de Tres de Febrero. Publicado en la República Argentina. 


\section{Introducción}

El libro de Charles Darwin El Origen de las Especies, introdujo la evolución por selección natural al ojo crítico del público en 1859. Pocas teorías se han esparcido más ampliamente y de forma tan rápida como lo hizo la teoría evolutiva de Darwin, no solo en la biología, sino también en la cultura (Browne 2009).

Aunque hay una laguna sorprendente en el estudio de la influencia del Darwinismo en el arte y en la cultura en general, cada vez hay más académicos que se hallan examinando dicha influencia en la literatura, en el arte y en la cultura visual popular. De modo que se puede decir que, uno de los aspectos más relevantes de los estudios actuales sobre la historia de la evolución, es el creciente interés por explorar su papel y alcance en ámbitos no científicos. En este sentido, el terreno de la educación ha cobrado gran relevancia. Sin embargo, son pocos los estudios realizados específicamente sobre el escenario mexicano.

Para entender el porqué de la relevancia de la teoría evolutiva en la educación, es necesario considerar su alcance y poder explicativo. En primer lugar, la propuesta formulada por Darwin en 1859 representa en la actualidad el pilar de la biología moderna, pues permite explicar los procesos, fenómenos y singularidades del mundo natural. Además, si la enseñanza de la evolución se sostiene en la historia y la filosofía de la ciencia, se promueve un mejor entendimiento de la naturaleza de la ciencia, de temas científicos específicos y sirve para generar cambios conceptuales al confrontar a los estudiantes con sus propias preconcepciones, creencias religiosas y otras visiones del mundo con explicaciones científicas y con el contexto histórico de descubrimientos científicos (Bybee 1991, Allchin 2003). También sirve para generar un pensamiento crítico que fomenta la toma informada de decisiones, lo cual es fundamental en (y para) cualquier democracia (UNESCO 2002). Por si fuera poco, la teoría evolutiva estableció que el vínculo entre todas las especies es de tipo genealógico (relaciones de ancestría-descendencia), lo cual tiene profundas implicaciones no solo biológicas, sino en cuanto a la percepción de los seres humanos hacia su entorno, sobre su naturaleza y acerca de sus propios orígenes. Por todo ello, el legado que Darwin dejó a partir del trabajo propio y de las propuestas previas de otros naturalistas (Lamarck 1809, Lyell 1831) ${ }^{1}$ dio pie al surgimiento y establecimiento formal de la biología en la segunda mitad del siglo XIX.

Para los interesados en el tema de la historia, la filosofía y la enseñanza de la ciencia surgen diversas interrogantes interesantes relacionadas con esta teoría. ¿Qué hace que una teoría científica cobre gran relevancia en la educación? ¿Cómo y dónde se pueden identificar rasgos de la recepción de la teoría evolutiva en la educación en México? ¿Qué aspectos de la teoría se han considerado relevantes en distintos periodos?

El presente artículo sugiere un nuevo enfoque historiográfico sobre la enseñanza de la evolución biológica en México durante la tercera década del siglo XX. Este enfoque toma en cuenta no solo el contexto nacional y regional, sino también el aspecto transnacional más relevante para el escenario mexicano durante este particular momento histórico.

El campo de investigación sobre la historia de la educación científica se ha expandido durante las últimas dos décadas, y las publicaciones han reconocido los cambios en relación con las narrativas, los protagonistas, los problemas, las fuentes y los marcos teóricos (Bertomeu-Sánchez 2015). Muchos autores han llamado la atención sobre los estudios transnacionales y han puesto de manifiesto una variedad de actores históricos desconocidos, cultura material, espacios, libros de texto y prácticas educativas que habían sido relegadas (Olesko 2006, Vicedo 2012). Estos nuevos trabajos buscan mostrar el contraste entre los contextos nacionales/locales y la perspectiva global o la universalidad de las narrativas tradicionales (Turchetti, Herran \& Boudia 2012).

\footnotetext{
${ }^{1}$ Darwin en su "bosquejo histórico" de la 3era edición de El Origen (Darwin 1861) menciona no menos de treinta predecesores importantes para él y su teoría.
} 
Este trabajo emplea algunos de los métodos, preguntas y teorías de la historia que afectan a la ciencia mundial para profundizar nuestra comprensión de la compleja circunstancia en la que se implementó la educación socialista en México y la apropiación de la educación por parte del Estado mexicano. Esto permite, además, situar la educación científica mexicana en un contexto más amplio. Lo anterior supone un desafío a las narrativas históricas tradicionales que enfatizan las diferencias entre los centros activos de producción y dispersión de ideas y prácticas científicas, y las periferias pasivas, y que han tendido a ignorar la perspectiva de los receptores. Debido a lo anterior, se verá, por ejemplo, que la génesis de la educación socialista mexicana se liga con los ideales de la Revolución Rusa, y con sus adaptaciones lógicas y naturales a las necesidades locales mexicanas en un período de gran inestabilidad política y de fuertes presiones sociales y económicas.

\subsection{El evolucionismo en México}

La teoría evolutiva ha sido determinante en la modificación de los planes y programas que conforman los libros de texto utilizados en las aulas de enseñanza del mundo occidental. Es claro que los cambios no son procesos inmediatos en los sistemas educativos globales. En cada país, región geográfica o comunidad, la nueva teoría se insertó en forma diferente. En este sentido el escenario mexicano es interesante, y ha sido estudiado por Genovés (1959), Maldonado-Koerdell (1959), Moreno (1984), Glick (1988) y Barahona (2009).

Los trabajos de estos autores indican que, en México, tal como ocurrió en Francia hubo un retraso en la introducción, difusión y aceptación del darwinismo. De acuerdo con Genovés, esto ocurrió principalmente por los conflictos armados que desestabilizaban al país, y de acuerdo con MaldonadoKoerdell (1959) el factor más importante fue el afrancesamiento mexicano de la época que exaltaba los ideales positivistas. No obstante, las discusiones acerca del evolucionismo no estaban ausentes en México, principalmente en las aulas universitarias y normales, así como en algunos grupos de académicos que mantenían correspondencia directa con colegas europeos atraídos por nuevas discusiones generadas en torno a la propuesta Darwiniana a finales del siglo XIX. Estas discusiones fueron importantes en el desarrollo y establecimiento de la biología moderna en México (Barahona 2009).

Resulta importante mencionar que la mayoría de los historiadores que han estudiado la manera en que las ideas de Darwin fueron recibidas en las distintas partes del mundo, se han apoyado en el concepto de la revolución darwiniana (véase por ejemplo Glick, Ruíz \& Puig-Samper 1999). No obstante, es fundamental considerar que la visión evolutiva que se volvió popular después de 1859 no fue estrictamente la de Darwin, sino aquella progresiva, rápida, ortogenética y lamarckiana (Hull 1988), que Peter Bowler en 1988 (Bowler 1988) bautizó como revolución no darwiniana. De forma que la historia debería ser contada como la "introducción del evolucionismo en México" y no como "la introducción del darwinismo en México", lo cual se hace evidente en el pluralismo evolutivo característico de finales del siglo XIX y principios del XX y en el que el darwinismo estricto no se sustenta (Ochoa \& Barahona 2016). De acuerdo con lo anterior, Moreno (1984) y Barahona (2009) coinciden en señalar al profesor Justo Sierra $(1848-1912)^{2}$ como uno de los primeros mexicanos que refieren el trabajo de Darwin y algunos conceptos de la teoría evolutiva en $1875 .^{3}$

En el caso de la educación superior, el naturalista francés radicado en México Alfredo Dugès (18261910), publicó en 1878 el texto Programa de un curso de zoología y en 1884 Elementos de zoología, los cuales mencionaban brevemente algunos aspectos principales de la teoría evolutiva. La historiografía tradicional sobre el evolucionismo en México ha mostrado a Dugès como un naturalista de la vieja escuela, quien expuso el darwinismo en sus escritos de manera somera. Moreno de los Arcos (1984, p. 33) argumenta, por ejemplo, que el naturalista francés "era un científico sereno, que no se dejó llevar

\footnotetext{
2 Positivista Spenceriano, profesor en la recién creada Preparatoria.

3 Esta mención ocurrió en una reunión de la Sociedad Metodófila Gabino Barreda y se publicó en la revista de dicha sociedad, en la cual J. Sierra destacó la importancia de la experimentación y observación como oposición a enfoques espiritistas, y señaló a la Teoría de la Evolución como la manera de explicar el origen de los seres organizados.
} 
por el entusiasmo en el asunto darwinista”. Por otro lado, a Dugés se le reconoce por presentar ideas ambiguas acerca de su aceptación hacia el evolucionismo en un sentido general. Sin embargo, esto resulta de una tradición historiográfica que supone que la tendencia de finales del siglo XIX era convertirse hacia el evolucionismo a través del darwinismo. Recientemente se ha propuesto otro tipo de enfoque basado en el reconocimiento de un pluralismo en las ideas desarrolladas en la biología del siglo XIX, en la cual el evolucionismo y el darwinismo no son más que visiones de posiciones periféricas (véase Amundson 1998, Ochoa \& Barahona 2009). Algunas de estas posiciones fueron el debate entre Georges Cuvier (1769-1832) con sus condiciones de existencia frente a la unidad de tipo de Étienne Geoffroy Saint-Hilaire (1772-1844), así como postulados como la recapitulación, concebida por Louis Agassiz (1807-1873), la ortogénesis de Edward Drinker Cope, y la morfología de Richard Owen (1804-1892), visiones protagónicas del momento, que moldearon la biología desde mediados del siglo XIX. De forma que los naturalistas del siglo XIX, como Dugès, interpretaban la forma orgánica a través de leyes naturales, una de las cuales era el evolucionismo. A pesar de ello, sin duda el trabajo de Dugés fue fundamental en la comprensión y enseñanza de la evolución en los albores del siglo XX en México.

En la medicina también se discutieron las ideas evolutivas. Por ejemplo, el Dr. José Ramírez (18521904), llevo a cabo un estudio sobre el "Origen teratológico de las variedades, razas y especies" (1878). En el que hace referencia a la propuesta de Darwin en un intento por rechazar el determinismo biológico del racismo. Los escritos de Ramírez fueron importantes en las discusiones médicas y evolutivas de su tiempo. Escribió que, para entender el origen teratológico y embriológico de las variaciones, razas y especies, era necesario considerar las leyes de la herencia y la adaptación, ya que sería a través de ellas que se lograría un entendimiento del fenómeno de la reproducción de los organismos vivos. Asimismo, consideraba la herencia y la evolución como fenómenos indivisibles, ya que la transmisión de características y la adaptación eran procesos vitales para la evolución. Como muchos de la época, Ramírez aceptaba la herencia de los caracteres adquiridos de la teoría de Lamarck, ya que éstas características producían adaptaciones de los organismos a su ambiente y, por lo tanto, la evolución de las especies.

Quizá fue Alfonso Luis Herrera (1868-1942), el personaje más destacado en cuanto a la introducción de la teoría evolutiva en la enseñanza de la biología. Generalmente se le atribuye como el principal introductor del darwinismo en México. No obstante, en sus escritos se aprecia claramente este pluralismo evolutivo ya mencionado, característico de principios del siglo XX. Su perspectiva evolutiva está influida por el materialismo presocrático, el pensamiento lamarckiano del filósofo Herbert Spencer, la filosofía romántica alemana, y la perspectiva embriológica de Ernst Haeckel (Ledesma 1992). Su texto Nociones de Biología (Herrera 1904) publicado en $1904^{4}$ para la cátedra de Biología General de la Escuela Normal para Profesores, fue fundamental para la enseñanza de la evolución en las escuelas normales, y por ende en las aulas de educación básica.

Sin embargo, independientemente de la presencia de tan importantes actores, que comprendían las cuestiones fundamentales de la teoría evolutiva y su posible impacto en la investigación biológica y en la enseñanza de las ciencias naturales, la teoría de la evolución -así como sus modificaciones posteriores- históricamente no ha sido considerada en verdad relevante para la investigación biológica mexicana y, por ende, para la enseñanza. Esto no quiere decir que la teoría haya estado completamente ausente de las aulas, pero, como iremos detallando a lo largo de este artículo, ha existido un tira y afloja respecto de la utilización de la teoría de la evolución en la instrucción de la biología, que responde principalmente a intereses políticos y a lo que Ludwick Fleck ([1934] 1979), denominó estilos $\mathcal{E}$ colectivos de pensamiento.

\footnotetext{
${ }^{4}$ En 1902, Herrera fundó la primera cátedra de Biología General en México, como sustitución a la de Historia Natural, en la Escuela Normal para Profesores. Para su enseñanza, escribió Nociones de Biología que se publicó en 1904.
} 


\section{El proyecto socialista en la educación mexicana}

Para hilar la trama del presente artículo desde una perspectiva transnacional, expondremos primeramente el contexto global en el que se insertó el proyecto socialista mexicano, para después enfocarnos en el ámbito local de apropiación de métodos e ideologías de enseñanza.

A principios del siglo XX, los estados emergentes neo-post-revolucionarios e independientes comenzaron a otorgar a la educación una nueva función: la de generar sentimientos de lealtad y unidad que condujeran a verdaderos estados-nación. Según Vázquez (1969), en México esta tarea se consideró esencial debido a la heterogeneidad de su población. Durante las primeras décadas del siglo $\mathrm{XX}$, las ideas de corte socialista influyeron notablemente en el pensamiento mundial, las cuales generaron expectativas de cambios económicos en la distribución de la riqueza. El modelo educativo que en Rusia desarrolló principalmente el pedagogo Antón Semiónovich Makarenko (1888-1939), sirvió como referente en la organización de los contenidos de los planes y programas de estudio mexicanos posrevolucionarios (por ejemplo, la importancia de la lectura como vía para la transformación de la conciencia colectiva, lo cual se aprecia en las campañas de alfabetización urbanas y rurales, en la organización de lecturas grupales y el desarrollo de materiales de apoyo). Lo anterior como intento por rescatar los ideales de la Revolución y por fortalecer las estrategias para la formación de los grandes sindicatos, con base en la idea de Makarenko del cambio social mediante la apropiación de las concepciones de bienes comunes y bienes sociales. Se aprecia, además, la influencia de la ideología socialista de la URSS en el mandato de cambio del artículo tercero constitucional, que detallaremos más adelante, pero que se refiere a la Educación y la ideología subyacente, ocurrido en 1933, con la subsecuente Reforma educativa de 1934 (Niebla 1985), así como en las demandas laborales que los movimientos obreros y sindicales exigían en México desde finales del siglo XIX.

Otra influencia global fueron los ideales ilustrados y positivistas. Según White (White \& Anchor 1979), el término "Ilustración" puede ser utilizado como sustantivo o adjetivo. Como sustantivo, la Ilustración designa la creatividad cultural sorprendente del período comprendido entre la Revolución Inglesa de 1688 y la revolución Francesa de 1789. Se utiliza como adjetivo cuando el término "denota una actitud específica de la mente" (White \& Anchor 1979, p. ix). Esta actitud se distingue más o menos por una creencia en que la razón humana, la ciencia y la educación son el medio principal para alcanzar una sociedad libre.

A mediados del siglo XIX, los ideales ilustrados se reconstituyeron con el término amplio de "liberalismo". Más tarde, en los albores del siglo XX, las creencias de la Ilustración en la razón, la ciencia y la educación se adoptaron fuertemente por muchos para hacer frente a los retos de las nuevas sociedades y culturas tecnológicas. Ideológicamente, es factible considerar el socialismo como uno de los herederos de la tradición de la Ilustración (Cheng 2009). El socialismo fue un fenómeno histórico mundial con unas dimensiones humanísticas complejas. En el escenario mexicano, el socialismo adoptó la forma de varias políticas de Estado, cuyo objetivo, extremadamente ambicioso y global, fue de remodelar la mente e incluso el carácter del pueblo mexicano para lograr el cambio tan necesario en un país principalmente rural y en su mayoría pobres. En este sentido, es posible apreciar algunas influencias de la iluminación en diversas políticas mexicanas de la década de 1930, ya que "el concepto de maleabilidad humana y la perfectibilidad se remonta a la tradición intelectual y política de Occidente desde la Ilustración” (Cheng 2009, p. 2).

\subsection{El contexto local}

El 4 de Julio de 1934 Lázaro Cárdenas asumió la presidencia de México. Poco antes, los diputados y senadores del Partido Nacional Revolucionario (PNR), en cumplimiento de los acuerdos de su convención, ya habían iniciado una reforma al Artículo 3o Constitucional para implementar un proyecto de educación socialista. Más allá de que esta política educativa con un contenido fuertemente ideológico fuera posible o no en el México de los treinta (capitalista), resulta fundamental considerarla 
como un intento real por cambiar de forma radical el sistema educativo mexicano para estimular y apoyar el proceso de cambio social que Cárdenas y otros veían tan necesario (Raby 1981), mediante la homogeneización de la educación en las comunidades rurales y urbanas, el fomento al pensamiento crítico y el combate a la superstición y el fanatismo religioso.

Cárdenas, al igual que otros revolucionarios, pensaba que, al desarrollarse el proyecto de la Revolución a plenitud, se llegaría a un sistema y a una sociedad socialistas, por lo que promovió fuertemente una conciencia nacionalista y progresista en la niñez mexicana, y buscó acercar la educación a las clases trabajadoras. En octubre de 1934 se aprobó una reforma al artículo 3o de la Constitución mediante la cual se instauraba la educación socialista en México. El artículo recién modificado señalaba:

La educación que imparta el Estado será socialista y además de excluir toda doctrina religiosa combatirá el fanatismo y los prejuicios para lo cual la escuela organizará sus enseñanzas y actividades en forma que permita crear en la juventud en concepto racional y exacto del universo y de la vida social.

Entre las finalidades de la escuela socialista, además de la obligatoriedad, gratuidad y laicidad, se contemplaba que ésta debía ser científica para brindar la capacidad de adquirir

una noción real del Universo, basada en los principios de causalidad y evolución, sujeta a las rectificaciones que el adelanto del conocimiento imponga, sin recurrir a las afirmaciones dogmáticas [...] haciendo que el acaso, la ignorancia y las supersticiones, dejen de ser reglas de conducta privada o colectiva (Cano 1941, p. 38).

Como pieza fundamental de esta importante transformación educativa, la Comisión Editora Popular, publicó nuevos libros de texto para las escuelas primarias del país. Se elaboraron dos series de libros para todos los grados escolares: la "Serie S.E.P.", para las escuelas primarias urbanas; y la serie "Simiente", para las escuelas rurales. Ninguna de estas series tuvo el carácter de obligatoria.

\subsection{El tema de la evolución biológica en los libros de texto durante la década de 1930}

Un aspecto interesante de la historia de la educación, es la manera en que las diferentes instituciones de enseñanza se transforman para satisfacer las prioridades nacionales en momentos determinados. En cada institución, el cambio tiene elementos particulares que es necesario separar analíticamente de aquellos generales que se derivan de sus propias trayectorias históricas y del entorno educativo. En este sentido, y dado que la teoría de la evolución comenzó a incluirse durante los últimos años del siglo XIX en los libros de texto de numerosos países, cabe preguntarse si la ideología socialista implicó un cambio importante en el enfoque de la enseñanza de las ciencias naturales en general, y del tema de la evolución biológica en particular, en el escenario mexicano.

Tradicionalmente, la botánica ha sido la disciplina de interés par excellence en México, aunque los trabajos zoológicos comenzaron a incrementarse bastante durante el siglo XIX. No obstante, no todos los elementos del estudio de las plantas y animales han recibido históricamente la misma atención, siendo los aspectos descriptivos y de clasificación los campeones en el monopolio del interés de nuestros científicos hasta bien entrado el siglo XX. De hecho, no fue sino hasta la década de 1930, cuando se puede considerar la verdadera existencia de instituciones mexicanas dedicadas a la investigación en biología, con la correspondiente presencia de revistas especializadas (i.e. Revista Mexicana de Historia Natural, establecida en 1939) (Piñero 1996). Se puede decir entonces que las décadas de 1930 y 1940 representan un periodo inicial de la profesionalización de la investigación biológica, y que ésta en su totalidad, fue extremadamente rezagada en hacer e incluir cuestionamientos relevantes sobre los seres vivos desde una visión evolutiva (que incluye sistemática, biogeografía, ecología, genética, fisiología, bioquímica, etcétera).

Respecto del tema educativo que nos interesa y también de acuerdo con Piñero, "la teoría evolutiva no ha sido una parte esencial en la enseñanza de la biología en México” (1996, p. 1). Pero, con base en el profundo cambio que supuso la denominada Escuela Socialista ¿el tema de la evolución fue considerado en los planes y programas de estudio? Y en caso afirmativo, ¿en qué materiales 
educativos y de qué forma? Cuestiones que buscamos responder en este artículo mediante la revisión de los planes y programas de estudio desde finales del siglo XIX y hasta 1940, pero principalmente aquellos que resultaron de la Reforma Educativa de 1934, así como por medio del análisis del contenido del tema de la evolución biológica en los libros de texto empleados durante este periodo.

$\mathrm{Al}$ revisar los mencionados planes y programas de estudio, es posible aseverar que el tema de la evolución biológica se incluyó en los libros de texto de primaria mexicanos en los últimos años del siglo XIX. Un ejemplo es el libro Conferencias científicas a los niños, de Julio S. Hernández publicado en 1898. No obstante, fue hasta la presidencia de Abelardo Rodríguez (1932-1934) y con la escuela socialista de los años treinta que el tema de la evolución biológica oficialmente se incluyó en los planes y programas de estudio de la educación de primera y segunda enseñanza. ${ }^{5}$ Estos planes y programas, se distinguen de los anteriores "cuyo punto de partida para el estudio de un grupo cualquiera, es la descripción monográfica de un animal típico", por enfatizar que lo más importante a tomar en consideración son "las características biológicas que van marcando en cada grupo la gradación admirable que entraña la evolución de los seres vivos” (Hernández 1898, p. 201). De forma que se aprecia un gran cambio conceptual en la enseñanza de las ciencias naturales en México a partir del proyecto socialista.

La ideología detrás de la enseñanza de la evolución biológica en este periodo, fue, por un lado, la formación de un espíritu crítico y científico en los ciudadanos que fomentara la racionalidad sobre la superstición y el dogma religioso, que promoviera una crítica de la visión única de pensamiento determinista; que vinculara la educación con la producción principalmente agraria y con las organizaciones populares y luchas sociales. Por otro lado, se buscó la transformación de la enseñanza de las ciencias naturales al permitir la libertad de cátedra, al presentar un método basado en leyes naturales para explicar el origen, la diversidad y la clasificación de los seres vivos (que implica el reconocimiento de tiempo profundo en la historia de la Tierra y de los seres vivos, la mutabilidad de las especies contraria al fijismo y el replanteamiento de la naturaleza del ser humano y su lugar en el Universo).

Es importante destacar que los libros de texto aprobados por la Secretaría de Educación Pública (SEP) durante este periodo, enfatizaban las posibles aplicaciones de la teoría evolutiva, en concordancia con un interés global por incluir la teoría en la enseñanza básica, pero en el particular contexto mexicano, en el cual el movimiento agrarista tenía gran peso político y social. En este contexto local, se buscaba instruir a los alumnos en el manejo de cultivos, cuáles eran las herramientas de domesticación y la importancia de la selección artificial en el sistema de producción agrícola. Lo anterior para brindar a los jóvenes mexicanos los conocimientos necesarios para el control eficaz y dominio de su entorno mediante principios biológicos organizados, tales como las leyes de la adaptación y la clasificación de las especies locales, así como el reconocimiento de su valor económico y de salud pública.

La introducción del tema de la teoría evolutiva en los libros de texto, además de poderse interpretar por la circulación e impacto de la ideología socialista en la enseñanza, se puede también entender como una consecuencia de la apropiación de ciertos conceptos evolutivos por parte de ciertos actores de la tradición zoológica y botánica de la época. Este grupo de científicos, herederos del legado de Alfonso L. Herrera, como Maximino Martínez o Enrique Beltrán, voltearon la mirada a la cultura material (colecciones de especímenes de plantas y animales) bajo un enfoque evolutivo, que se reflejó en la incorporación de una interpretación de los seres vivos a través del tiempo en algunos libros de texto (Beltrán et al. 1952). No obstante, es interesante señalar que la influencia de ciertos actores históricos en el devenir de tradiciones y programas de investigación puede resultar determinantes en la ciencia y que lo que llamamos 'hechos', de acuerdo con Fleck ([1934] 1979) son constructos sociales, es decir que solo lo que es cierto para la cultura es cierto para la naturaleza. En México, las preguntas fundamentales de la evolución biológica no fueron realmente aplicadas al estudio de los seres vivos sino hasta hace pocas décadas, principalmente porque el primer director del Instituto de Biología de la

\footnotetext{
5 Desafortunadamente, no se han encontrado materiales educativos relacionados con la enseñanza de la teoría evolutiva en libros de texto de educación básica durante el México postrevolucionario de 1910 a 1930.
} 
Universidad Nacional Autónoma de México (primero y único en su tipo) fundado en 1929 fue Isaac Ochoterena. Siguiendo la propuesta de Fleck de estilos y colectivos de pensamiento, Ochoterena empujó tanto los intereses como los métodos de la investigación biológica hacia la parte más descriptiva y clasificatoria de la historia natural: la catalogación de los recursos biológicos de México. Es por ello que, durante buena parte del siglo XX, hubo "una especie de inercia académica fuera de un contexto evolutivo" (Piñero 1996) en esta fundamental y única institución dedicada a la investigación biológica, por lo que se puede generalizar a todo México. Veremos más adelante que esto explica, en parte, la razón subyacente a la reducción del tema de la teoría evolutiva en la enseñanza y en los libros de texto mexicanos de 1940 a 1999.

\subsection{1 ¿De qué forma se abordó el tema de la evolución en los libros de texto de la escuela socialista?}

Ahora bien, con relación a la cuestión ¿en qué materiales educativos y de qué forma fue incluida la teoría la evolutiva?, podemos decir que en la educación primaria se enseñaban distintos temas relacionados con la evolución desde cuarto año (segundo grado del segundo ciclo, en la terminología de la época). El libro de texto de actividades Para Conocer la Naturaleza de cuarto año de primaria contemplaba el estudio del origen y evolución de los animales en la materia de zoología (Cano 1941, p. 147) y en el segundo grado del tercer ciclo ( $6^{\circ}$ año), el origen y la evolución de las plantas en la materia de botánica. En general, el contenido que los libros de texto presentaban sobre el tema, era reducido y abordado de forma somera. Se presentaban las generalidades de los seres vivientes en función de su origen y evolución, su utilidad, su estructura, su adaptación al medio y su clasificación.

Referente a los programas y planes de estudio de segunda enseñanza, en la descripción de las finalidades pedagógicas de Ciencias Biológicas destaca lo siguiente: "Poner al alumno en contacto con la naturaleza a fin de iniciarlo en el estudio de la vida, la interpretación de las leyes biológicas y la evolución de los organismos" (Cano 1941, p. 201).

Es interesante resaltar que la enseñanza de las ciencias biológicas en secundaria durante la década de 1930 se dividía en botánica en primero, zoología en segundo y anatomía, fisiología e higiene en tercero (similar a los textos de Civic biology de la enseñanza estadounidense). Cada disciplina ejemplificaba detalladamente la anatomía, fisiología y clasificación de los grupos representados. Un texto fundamental tanto por el número de ediciones como por el tiraje fue Guía para el curso de Zoología (1939) de Maximino Martínez cuyo último capítulo está dedicado al tema de la Evolución. Es relevante que el autor precisa en la introducción del capítulo lo siguiente:

Vamos a señalar brevemente los lineamientos generales de esta teoría siguiendo a diversos autores, advirtiendo que no es nuestra mente imponerla, sino exponer los hechos sujetándolos a un examen libre que lleve a la conclusión que racional y lógicamente se desprenda de ellos (Martínez 1939, p. 276).

El capítulo aporta diversas evidencias a las que refiere como hechos de la anatomía, paleontología, embriología y de reacciones humorales. En un apartado menciona las causas de la variabilidad de las especies y las explicaciones dadas por Darwin, Lamarck, Geoffroy de St. Hilare y Hugo de Vries. Curioso es el hincapié que hace Martínez que de la teoría evolutiva no se puede inferir que el mono engendre al hombre, sino más bien que éste deriva de un tipo primitivo o "eslabón perdido" que relaciona a la especie humana con especies inferiores $(1939$, p. 284).

\subsection{La desaparición de la educación socialista y del tema de la evolución biológica en 1940}

Debido a la poca claridad del proyecto de la educación socialista mexicana, la SEP creó en 1937 el Instituto de Orientación Socialista. La finalidad de este instituto consistía en fortalecer la comprensión de los maestros acerca del significado de diversos temas fundamentales para la filosofía educativa socialista, tales como la naturaleza de la explicación científica, las definiciones de disciplina colectiva y socialismo científico y el impulso en la unión del proletariado. Lo anterior bajo el supuesto de que para 
el aprendizaje de la biología era importante combatir fanatismos, prejuicios, errores conceptuales y supersticiones (AGN 1935).

Aun así, el proyecto de educación socialista finalizó en 1940. Las razones de tan corta duración son profundas y complejas puesto que involucran situaciones políticas y económicas tanto globales como locales que exceden los objetivos del presente texto. No obstante, es necesario señalar que las instituciones educativas -de la naturaleza que sean- representan uno de los principales mecanismos del Estado para conservar el poder. Resulta interesante notar que estas instituciones, y la forma en la que están organizadas y controladas, representan acceso a recursos económicos, culturales e ideológicos para un número limitado de personas, pero al mismo tiempo pertenecen a la esfera pública regulada por el Estado. De forma que una vez finalizado el sexenio de Cárdenas, su proyecto educativo, único en su tipo, no fue defendido, en parte por una ausencia de claridad teórica (Raby 1981, p. 82).

En 1940 Manuel Ávila Camacho asumió la presidencia de México. Octavio Véjar Vázquez, segundo secretario de educación pública de aquel sexenio en 1941, quien se manifestó en contra de la escuela socialista y en favor de la escuela nacional, lo cual implicó la remoción de cualquier libro con tintes comunistas. Con el fin de oficializar el cambio de materiales educativos, el gobierno de Ávila Camacho, publicó el 2 de febrero de 1941 una nueva lista con los textos que habían sido aprobados por el consejo de escuelas primarias del Distrito federal para escuelas primarias y rurales. De éstos, muy pocos mencionaban el tema de la evolución, por ejemplo, los libros de Naturaleza y ciencias, escrito para el sexto año de las escuelas primarias de R. Jáuregui (1955) y Academias. Estudio de la naturaleza para uso del $5^{\circ}$ grado de enseñanza primaria de Irene Motts e Imelda Calderón (1954).

Fue en 1946, bajo la presidencia de Miguel Alemán cuando se reforma nuevamente el Art. 3 y se elimina el término "Socialista", autorizando a particulares a impartir la educación (aunque seguiría siendo supervisada por el estado).

\section{Conclusiones}

El contexto nacional e internacional, generalizadamente convulsivo durante las primeras décadas del siglo XX, imprimió características particulares de intensidad e intolerancia a los debates en relación con la reforma educativa mexicana de 1930, que aconteció en el marco del proyecto socialista mexicano. En el ámbito de la educación, este proyecto, representa uno de los principales esfuerzos del estado mexicano para proporcionar una educación masiva e incluyente, el cual, independientemente de su corta vida, sirvió como modelo de proyectos educativos tan importantes como el cubano.

A diferencia de otros proyectos educativos, la propuesta socialista reconocía a los trabajadores como motores de cambio social, y resultaba muy atractiva como proyecto educativo ante las condiciones de pobreza y analfabetismo característico del momento. ${ }^{6}$

Como mostramos en este trabajo, durante el México postrevolucionario era necesario encauzar la educación pública a todos los niveles y sectores de la ciudadanía. La educación socialista buscaba desarrollarse a través de la vinculación con organizaciones populares activas, con la demanda productiva que la nación necesitaba, y como un vehículo de propaganda y difusión de la política gubernamental. Fue particularmente importante el papel que jugaron las formaciones culturales de izquierda en la elaboración de los planes de estudio y los libros de texto bajo la suposición de un desplome capitalista y con la esperanza de un nuevo orden social que contribuyera a un cambio significativo en la ideología que se pensaba indispensable para la ciudadanía.

Bajo los preceptos socialistas, el papel de la escuela moderna de 1930, era la de formar hombres y mujeres con un pensamiento crítico y racional, liberados de ideologías, fanatismos, y que respetaran los derechos y deberes de los individuos. De ahí que la temática y contenidos de los libros de texto se

\footnotetext{
${ }^{6}$ En 1930, México era principalmente rural y con altos índices de pobreza extrema, con una población cercana a los 16 millones. El crecimiento económico era negativo, el desempleo se había agravado por el regreso a México de más de 70,000 trabajadores que se encontraban en Estados Unidos debido a la crisis de 1929. La tasa de analfabetismo era cerca del 65\% de la población.
} 
encauzara a situaciones sobre la granja, el taller, el laboratorio, la fábrica, es decir en espacios comunitarios de enseñanza.

Los planes y programas de estudio se transformaron de acuerdo a las necesidades del campo y la ciudad, lo que se reflejó en los libros de texto y en los métodos de enseñanza que buscaban cumplir los ideales socialistas. Se buscaba incorporar, además, a sectores de campesinos, obreros y otros trabajadores, con el fin de fortalecer una formación social y abatir el analfabetismo tan marcado de la época. Como se explicó anteriormente, la circulación de nuevas estrategias pedagógicas (como las de Makarenko y Ferrer) contribuyeron a la reflexión de las necesidades que requería la nueva propuesta de nación.

En el ámbito de la enseñanza de la teoría evolutiva, la inclusión del tema a nivel nacional fue sin duda una de las experiencias educativas más singulares que ocurrieron en América Latina en el primer tercio del siglo XX. El análisis historiográfico de los textos de enseñanza, materiales y pronunciamientos emitidos por el estado durante este periodo, contundentemente muestran como en México durante la década de 1930 se incluyen temas de la evolución biológica en los planes y programas de estudio de la educación primaria y secundaria y en los libros de texto correspondientes, ocasionando una evidente ruptura con las tradiciones pedagógicas de otros momentos de la escuela mexicana posrevolucionaria.

El nuevo carácter pedagógico se sustentaba en evidencias científicas que buscaban mostraban con claridad las propuestas sobre el origen de la vida, la transmisión de generación en generación, así como nociones fundamentales de los procesos de los seres vivos que emanciparían a los niños de prejuicios, errores y supersticiones.

Este cambio conceptual y profundo buscaba contribuir en el desarrollo de una formación crítica aislada de fanatismos o prejuicios que la ideología religiosa había promovido e incluido desde el inicio de la conquista.

Este proyecto educativo sin duda generó tensiones en los diferentes sectores de la sociedad mexicana que de inmediato se opusieron y protestaron en contra de la propuesta promovida por Lázaro Cárdenas. La Reforma Educativa de 1934 no tuvo un desarrollo homogéneo, dado que los lineamientos oficiales contenidos en el plan de acción de la escuela socialista, fueron adaptados en función de tradiciones locales y recibieron respuestas distintas por parte de las comunidades. Las labores de los maestros fueron mediatizadas por las dinámicas de cada población y por las disputas entre los diferentes grupos y corrientes que intervinieron en estas luchas.

Es claro que resultó ingenua la idea de que se podía realizar un cambio de conciencias por medio de un decreto. Los libros de texto escolares, los panfletos y la propaganda en general se multiplicaron, aunque muchos maestros se quedaron desfasados para entender plenamente en qué consistía la Reforma educativa a profundidad. Formar a un niño para la vida posrevolucionaria, resultaba complejo para los maestros, quienes no contaron con el tiempo suficiente para apropiarse la propuesta.

Sin duda, el gobierno de Cárdenas intentó llenar con propaganda socialista el entorno escolar, lo cual no fue suficiente para modificar las prácticas y modelos pedagógicos de muchos profesores rurales. Incluso se promovió un Instituto de Orientación Socialista en 1937, para reforzar la idea de una "explicación socialista" y un "socialismo científico", que buscaba incidir en la formación de las nuevas generaciones de niños libres de prejuicios, con una visión crítica de su entorno que rescataba los ideales de la ilustración y la ciencia.

Finalmente, al tomar posesión Ávila Camacho en 1940, la educación socialista fue modificada, adaptándola a un modelo de corte nacionalista. 
Academia Mexicana de Jurisprudencia y Legislación (1934), La proyectada reforma del artículo $3^{\circ}$ de la Constitución: opinión de la Academia de Jurisprudencia y Legislación, correspondiente de la España acerca de la enseñanza socialista, México: Talleres gráficos Laguna.

AGN (1935), Sep Archivo, México: Instituto de Orientación Socialista.

Allchin, D. (2003), “Scientific Myth-Conceptions”, Science Education 87(3): 329-351.

Amundson, R. (1998), “Typology Reconsidered: two Doctrines on the History of Evolutionary Biology”, Biology $\mathcal{E}$ Philosophy 13: 153-177.

Barahona, A. (2009), "Introducción del Darwinismo en México”, Teorema 28(2): 201-214.

Beltrán, E., Rioja, E., Alcaraz, J., Ruiz, M., Miranda, F. e I. Larios (1952), Biología. Segundo curso para escuelas secundarias, México: Editorial Porrúa.

Bertomeu-Sánchez, J.R. (2015), “Beyond Borders in the History of Science Education”, en Arabatzis, T., Renn, J. y A. Simões (eds.), Relocating the History of Science, Dordrecht: Springer, pp. 159-173.

Bowler, P. (1988), The Non-Darwinian Revolution: Reinterpreting a Historical Myth, Baltimore, MD: The Johns Hopkins University Press.

Browne, J. (2009), “Looking at Darwin: Portraits and the Making of an Icon”, Isis 100(3): 542-570.

Bybee, R. (1992), Evolution in Perspective: The Science Teacher's Compendium, Arlington: National Science Teachers Association (NSTA) Press.

Cano, C. (1941), La educación pública en México desde el 1 de diciembre de 1934 hasta el 30 de noviembre de 1940, México: Talleres Gráficos de la Nación.

Cheng, Y. (2009), Creating the New Man: From Enlightenment Ideals to Socialist Realities, Honolulu: University of Hawaii Press.

Darwin, C. (1859), The Origin of Species, $1^{\text {st }}$ edition, London: Murray.

Darwin, C. (1861), The Origin of Species, $3^{\text {rd }}$ edition, London: Murray.

Dugés, A. (1878), Programa de un curso de zoología, México: Justo Palencia.

Dugés, A. (1884), Elementos de Zoología, México: Oficina Tipográfica de la Secretaría de Fomento.

Fleck, L. ([1934] 1979), Genesis and Development of a Scientific Fact, Chicago: University of Chicago Press.

Genovés, S. (1959), "Darwin y la antropología”, Revista de la Sociedad Mexicana de Historia Natural 20: 31-41.

Glick, T.F. (ed.) (1988), The Comparative Reception of Darwinism, Chicago: University of Chicago Press.

Glick, T.F., Ruiz, R. y M.A. Puig-Samper (eds.) (1999), El darwinismo en España e Iberoamérica, Madrid: Editorial CSIC.

Hernández, J.S. (1898), Conferencias científicas a los niños, México: Lib. Madrileña de J. Buxo y Com.

Herrera, A. (1904), Nociones de Biología, México: Secretaria de Fomento.

Hughes, L. (1951), Las misiones culturales mexicanas y su programa, París: UNESCO.

Hull, D.L. (1988), "Progress in Ideas of Progress", en Nitecki, M.H. (ed.), Evolutionary Progress, Chicago: University of Chicago Press, pp. 27-48.

Jáuregui, R. (1955), Naturaleza y ciencias. Libro 5o., escrito para el sexto año de las escuelas primarias, México: Editorial Patria.

Lamarck, J.B.P. (1809), Philosophie zoologique, Paris: Lamb Frye.

Ledesma, I. (2002), "La introducción de los paradigmas de la biología en México y la obra de Alfonso L. Herrera”, Historia mexicana 52(1): 201-240. 
Lyell, C. (1831), "Reply to a Note in the Rev. Mr. Conybeare's Paper entitled 'An Examination of those Phænomena of Geology, which seem to bear most directly on theoretical Speculations”, The Philosophical Magazine 9(49): 1-7.

Maldonado-Koerdell, M. (1959), "Linneus, Darwin y Wallace en la bibliografía mexicana deficiencias naturales. I. Primeras referencias a sus ideas en México", Revista de la Sociedad Mexicana de Historia Natural 20: 63-78.

Martínez, M. (1939), Zoología, México: Editorial Botas.

Moreno, R. (1984), La Polémica del darwinismo en México, siglo XIX: testimonios, Vol. 1, México: Universidad Nacional Autónoma de México. Estudio de la Naturaleza para uso del $5^{\circ}$ grado de ense

Motts, I. e I. Calderón (1954), Estudio de la Naturaleza para uso del 5º grado de enseñanza primaria, 4a edición, México: Imprenta Aldina Rosell y Sordo Noriega, S.R.L.

Niebla, G. (1985), La educación socialista en México (1934-1945), México: SEP.

Ochoa, C. y A. Barahona (2009), Forma versus función: Historia de la homología y la analogía, México: Universidad Nacional Autónoma de México.

Ochoa, C. y A. Barahona (2016), "La introducción del evolucionismo en México a través de los libros de texto de biología: Alfredo Dugès, Alfonso L. Herrera, Isaac Ochoterena y la revolución no darwiniana”, en Torrens, S. y A. Barahona (eds.), La introducción del evolucionismo en México, México: Universidad Nacional Autónoma de México, en prensa.

Olesko, K.M. (2006), "Science Pedagogy as a Category of Historical Analysis: Past, Present, and Future”, Science E⿱ Education 15(7-8): 863-880.

Piñero, D. (1996), “La teoría de la evolución en la biología mexicana: una hipótesis nula”, Ciencias 40: 13-17.

Raby, D.I. (1981), “La educación socialista en México”, Cuadernos Políticos 29: 75-82.

Ramírez, J. (1878), “Origen teratológico de las variedades, razas y especies”, La Naturaleza (1877-1879) IV: 235-247.

Turchetti, S., Herran, N. y S. Boudia (2012), "Introduction: Have We Ever Been 'Transnational'? Towards a History of Science Across and Beyond Borders", The British Journal for the History of Science 45(3): 319-336.

UNESCO (2002), “Teaching and Learning for a Sustainable Future". Accesible en: http://www.unesco.org/education/tlsf/mods/theme_d/mod20.html.

Vázquez, J. (1969), “La educación socialista de los años treinta”, Historia Mexicana 18(3): 408-423.

Vicedo, M. (2012), "Introduction: The Secret Lives of Textbooks", Isis 103(1): 83-87.

White H.V.y R. Anchor (1979), The Enlightenment Tradition. Introduction, California: University of California Press. 